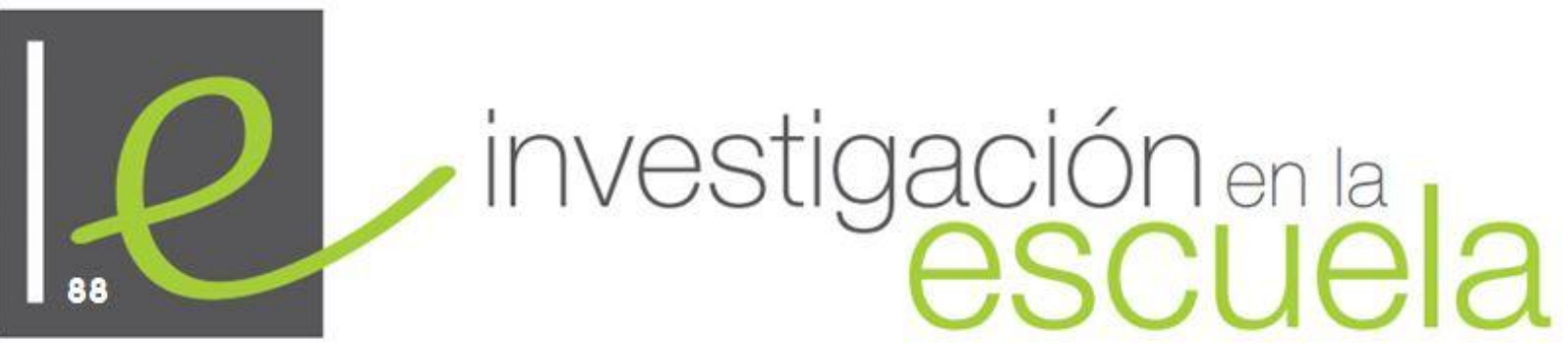

Revista académica evaluada por pares y de acceso abierto

\title{
Los trabajos por proyectos en la Educación Infantil. En primera persona: estudio de caso biográfico-narrativo de una maestra.
}

\author{
Pablo Javier Ortega Rodríguez. \\ Universidad de Huelva \\ España
}

Citación: Ortega, P. (2016). Los trabajos por proyectos en la Educación Infantil. En primera persona: estudio de caso biográfico-narrativo de una maestra. Investigación en la Escuela, 88, 112-126. Recuperado de www.investigacionenlaescuela.es/artiuclos/R88/R88-7

Resumen: el trabajo por proyectos, entendido como un modelo alternativo para el desarrollo del currículum, se basa en el establecimiento de relaciones entre diferentes áreas de conocimiento, con la finalidad de abordar una cuestión a través de un aprendizaje significativo. A partir de una investigación cualitativa, se ha analizado el relato de vida de una maestra de Educación Infantil y el punto de vista del equipo docente de un centro educativo, mediante la realización de un ciclo de entrevistas biográfico-narrativas y la elaboración de un inventario de declaraciones. El artículo concluye que los trabajos por proyectos requieren de un grupo sólido de maestros y maestras, dispuestos a profundizar en las temáticas del currículum mediante el planteamiento de problemas socialmente relevantes, partiendo de los aspectos ligados a la vida cotidiana de los niños y las niñas. El aprendizaje profesional de la profesora no es una mera transferencia de los saberes individuales implícitos en su práctica cotidiana, sino el resultado de la interacción con otros agentes educativos y experiencias que facilita la reflexión sobre su trayectoria profesional y personal.

Palabras clave: "trabajo por proyectos"; "currículum integrado"; "educación infantil"; "historia de vida"; "método biográfico-narrativo". 
The project based learnign in childhood education. In first person: case study biographical-narrative of a teacher

Abstract: project work, understood as an alternative model for curriculum development, is based on the establishment of relationships between different areas of knowledge, with the aim of addressing an issue through significant learning. From a qualitative research, the life story of a kindergarten teacher has been analyzed and the point of view of an educational institute's teaching staff, through a series of biographical narrative interviews and the development of an inventory of statements. The article concludes that project work requires a solid group of teachers, willing to deepen their understanding in the area of the curriculum, through the approach of socially relevant problems, based on aspects linked to the daily lives of children. Professional apprenticeship from the teacher is not a mere transfer of implicit individual knowledge into daily practice, but it's the result of the interaction with other educational agents and experiences which facilitates the reflection on one's professional and personal career path.

Key words: "project work"; "integrated curriculum"; "childhood education"; "life history"; "narrative biographical method".

Les travail par projet dans l'enseignement préscolaire. À la première personne: études de cas biographique-narratif d'une enseignante

Resumé: le travail de projets, en tant que modèle alternatif pour développer le programme d'études, est basé sur l'établissement des relations entre les différents domaines de la connaissance, afin d'aborder une problématique par le biais d'un apprentissage significatif. À partir d'une recherche qualitative, le cursus d'une professeure des écoles et le point de vue de l'équipe pédagogique d'un établissement d'enseignement ont été analysés, grâce à une série d'entretiens biographiques et narratifs et de l'élaboration d'un inventaire des déclarations. L'article conclut que les travaux de projets nécessitent un groupe de professeur(e)s motivés et disposés à approfondir les thèmes du programme d'études en soulevant des questions sociales pertinentes, relatives à la vie quotidienne des enfants. L'apprentissage professionnel de l'enseignant ne consiste pas seulement en une transmission des connaissances individuelles implicites dans leur pratique quotidienne; c'est aussi le résultat d'expériences et de l'interaction avec d'autres agents éducatifs, qui leur permet de réfléchir à leur parcours professionnel et personnel.

Mosts clé: "travail de projets"; "programme d'etudes intégrant plusieurs niveaux"; "maternelle"; "cursus"; "méthode biographique et narrative"

\section{Introducción}

El trabajo por proyectos es un modelo alternativo al enfoque curricular tradicional, basado en unos principios pedagógicos fundamentados, coherentes y sólidos, derivados de diferentes fuentes y perspectivas. Esta hibridación de fundamentos en los que se sustenta son los siguientes: el aprendizaje significativo, el enfoque cooperativo, la investigación sobre la práctica y las perspectivas crítica y comunitaria (Díez, 2002; Pozuelos, 2007). El enfoque cooperativo convierte el aula en un espacio de diálogo, reflexión y discusión entre los grupos heterogéneos de alumnos y alumnas, de manera que cotejando diferentes puntos de vista, pueden analizar y valorar las diversas soluciones posibles (López Melero \& Parages López, 2012). Como sugiere Guarro (2005, p. 24) este enfoque se puede bifurcar en dos direcciones: como forma de convivencia democrática y como modelo de enseñanza aprendizaje. Se puede entender la cooperación como una oportunidad para favorecer la socialización, la libre participación y la interacción entre niños y niñas, surtiendo efectos positivos en 
el aprendizaje de habilidades sociales con vistas a trabajar en grupo el respeto y la creación de las normas de convivencia.

La investigación sobre la práctica consiste en una sucesión de actividades que conforman un proceso de búsqueda de información, comprensión, reflexión compartida y respuesta ante diferentes preguntas planteadas. El trabajo por proyectos es un enfoque basado en la investigación, cuya proyección va más allá del carácter incuestionable de los contenidos del libro de texto. Como refiere Martínez Bonafé (2002, p. 23): “el signo del libro de texto muestra un orden experiencial del discurso pedagógico donde permanecen inalterables las respuestas a las preguntas: de qué se habla y cómo se habla en la práctica institucional". Se basa en el establecimiento de relaciones entre diferentes áreas de conocimiento, con la finalidad de abordar una cuestión o resolver un problema a través de un aprendizaje globalizado y significativo (Hernández \& Ventura, 2008). El aprendizaje a través de la investigación conlleva la participación del alumnado en distintas experiencias que suscitan la motivación, el interés y la adquisición de conocimientos basada en la comprensión, poniendo en duda la perspectiva adquirida hacia una cuestión, abriendo nuevos cauces de indagación: formular preguntas, hallar información, verificar los datos y obtener conclusiones (Pozuelos, 2007). El punto de vista crítico en los trabajos por proyectos ofrece la oportunidad de adoptar un enfoque más neutral, sin la intencionalidad oculta en los libros de textos, garantes de una cultura académica, que en ocasiones silencian un conjunto de voces pertenecientes a los grupos sociales minoritarios: el mundo femenino, las etnias minoritarias, el mundo rural y marinero, la clase trabajadora, entre otras (Torres, 2012). Cultura que se corresponde con un conjunto de disciplinas escolares, aglutinadas en manuales que pretenden instaurar en el alumnado una determinada interpretación de la realidad y un significado intencionado mediante el aprendizaje memorístico (Pérez, 1998).

Desde esta óptica crítica, se contribuye a estimular el pensamiento crítico de los discentes ante temas como el racismo, la injusticia y otras desigualdades de la sociedad y de los centros educativos (Apple \& Beane, 2005). No se ha de pasar por alto que la escuela es un reflejo de las condiciones de la sociedad, razón por la cual es esencial conectar la formación impartida dentro del contexto escolar con el aprendizaje derivado de aquellos sectores exteriores: la implicación de las familias, los recursos de diferentes instituciones y las visitas externas al centro (Feito, 2006).

Desde un punto de vista sociocrítico, la educación es un fenómeno complejo, que ocurre en un contexto donde existen muchos participantes (profesorado, alumnado, familia, diferentes sectores de la comunidad) que interaccionan. Este principio contribuye a desvelar el significado de sus valores, creencias y actitudes ante todos los hechos que acontecen en la realidad educativa (Pérez Gómez, 1998). Este hecho se refleja de manera más evidente en los estudios biográficos, puesto que cada persona proporciona una experiencia concreta e irrepetible. El estudio de una persona como caso ofrece la posibilidad de conocer su experiencia, sus acciones, comprendiendo sus actitudes y aportando nuevos significados a una situación o tema general. En este sentido, Day (2006) sostiene que el análisis de la vida personal y profesional, y de las historias laborales sirven como acicate para comprender los orígenes que sustentan las creencias, los pensamientos y las prácticas mantenidas actualmente por el/la docente, permitiendo conocer las influencias que las han conformado.

El estudio de caso biográfico-narrativo es el método más idóneo para conocer la vida personal y profesional del profesor, de la profesora, sirviendo como retroalimentación para su desarrollo docente. Además, brinda la oportunidad de explorar, describir y explicar cualquier temática a partir de una experiencia personal, poniendo de manifiesto nuevos significados y perspectivas sobre la misma, ayudando a comprender otros casos similares (Bolívar, Domingo, \& Fernández, 2001). También concibe al docente como un profesional que aprende de forma permanente, en interacción con un conjunto de contextos y de personas que le invitan a reflexionar sobre el entorno en el que trabaja, su forma de actuar, los motivos que han condicionado su 
personalidad docente, brindándole la posibilidad de comprender el origen de su pensamiento, sus acciones y su estilo pedagógico. Salgueiro (1998, p. 38) refiere que "el trabajo docente es el resultado de la interacción con otros docentes, con especialistas, con el alumnado y con sus familias, además de un proceso colectivo de elaboración, de reflexión, articulado desde lo individual".

\section{Metodología}

El marco empírico de la presente investigación cualitativa emplea el método de estudio de caso biográfico-narrativo, con la finalidad de analizar, profundizar y comprender la historia de vida de una maestra de Educación Infantil. A su vez, es un caso inclusivo, porque el estudio incluye la investigación de subunidades (grupo de maestras de la misma etapa) para contrastar los resultados del caso principal, contribuyendo a la comprensión global de la historia de la docente (Buendía, Colás, \& Hernández Pina, 1998). Su carácter intrínseco deriva de su interés por el aprendizaje que reporta al tema general: la educación intercultural a través del trabajo por proyectos. El método biográfico-narrativo se circunscribe a la epistemología interpretativa-hermenéutica, que se traduce en una interacción entre el investigador y el narrador, con el objetivo de que éste estructure su vida en relación con otras personas, espacios y tiempos (Bolívar, Domingo, \& Fernández, 2001). La reciprocidad entre ambos ha favorecido la construcción de los significados compartidos sobre los acontecimientos que marcan la experiencia narrada (Simons, 2011).

Los objetivos que se han propuesto fueron los siguientes:

a) Conocer el relato de vida de una docente, cuya trayectoria profesional se articula en torno a los proyectos de trabajo sobre la educación intercultural, comprobando cómo su experiencia contribuye a una nueva interpretación de esta dinámica.

b) Construir la biografía evolutiva de la maestra, identificando las influencias, los incidentes críticos, sus reflexiones, los significados atribuidos a los acontecimientos personales y el impacto de otras vidas en su práctica docente.

c) Interpretar el testimonio subjetivo de la docente a partir de las circunstancias familiares, personales, políticas, sociales, culturales y pedagógicas que han marcado su vida.

d) Estudiar en profundidad la relación entre los proyectos de trabajo y la interculturalidad desde la perspectiva biográfico-narrativa de la maestra, descubriendo significados particulares del relato y las aportaciones idionsicrásicas que éstos reportan.

e) Describir la valoración personal del equipo docente sobre las declaraciones de la maestra acerca del proceso de enseñanza-aprendizaje en Educación Infantil.

f) Explicar las expectativas y las perspectivas futuras de la maestra en relación a los proyectos de trabajo en su nueva proyección profesional.

\section{Descripción del caso}

La maestra imparte docencia en el Colegio Público de Educación Infantil y Primaria Virgen del Carmen, ubicado en el centro del municipio de Punta Umbría, perteneciente a la provincia de Huelva. El equipo de ciclo de Educación Infantil, seis maestras, también ha sido protagonista del presente estudio. Cuatro de ellas tienen plaza definitiva en el centro, una es interina y otra, provisional. Todas han tenido oportunidad de tomar contacto con los trabajos por proyectos, bien por la relación directa con su estilo de enseñanza, bien por el conocimiento de experiencias procedentes de sus compañeras. Las profesionales más veteranas tratan de orientar a las nuevas maestras, explicándoles la metodología de trabajo característica del centro y la estrecha colaboración familia-equipo docente. 


\section{Recogida y análisis de datos}

En esta investigación, se ha optado por la utilización de un amplio abanico de instrumentos cualitativos para la recogida de datos, con vistas a alcanzar los objetivos planteados anteriormente:

a) Ciclo de entrevistas biográfico-narrativas. Es un instrumento de investigación que ayudó a la maestra a reconstruir su vida, a partir de categorías temáticas relacionadas con su trayectoria personal y profesional (Kvale, 2011). Consistió en una secuencia de tres entrevistas que se realizó en diferentes momentos. La primera se correspondió con el diseño de un protocolo semi-estructurado de 31 cuestiones amplias, clasificadas en cuatro bloques temáticos en orden cronológico (fase inicial, época de tránsito al trabajo estable, primeras experiencias, fase de madurez /desarrollo profesional, proyección de futuro). Se procedió a realizar la primera transcripción y posterior análisis con una doble finalidad: realizar una primera reconstrucción de su vida para identificar puntos oscuros o aspectos inconexos, vagos o poco desarrollados. La segunda entrevista estuvo orientada a la búsqueda de respuesta a estos aspectos identificados previamente, abordando temas relacionados con la experiencia profesional, a saber, primeras experiencias docentes con los trabajos por proyectos, formación permanente e influencias de personas importantes. Se puso especial énfasis en los incidentes críticos, es decir, aquellos acontecimientos (cambio de centro educativo, circunstancias familiares, experiencias significativas) que marcaron un punto de inflexión en la trayectoria de la maestra y conllevaron un impacto en su desarrollo profesional. Posteriormente, se efectuó un análisis (transcripción e interpretación) para obtener un conjunto de conclusiones, confeccionando una tabla de categorías. La tercera entrevista sirvió para debatir con la maestra las afirmaciones que había extraído de su relato, reflexionar sobre aquellos argumentos convenientes y negociar significados sobre la interpretación de sus declaraciones (ver Gráfico 1). 


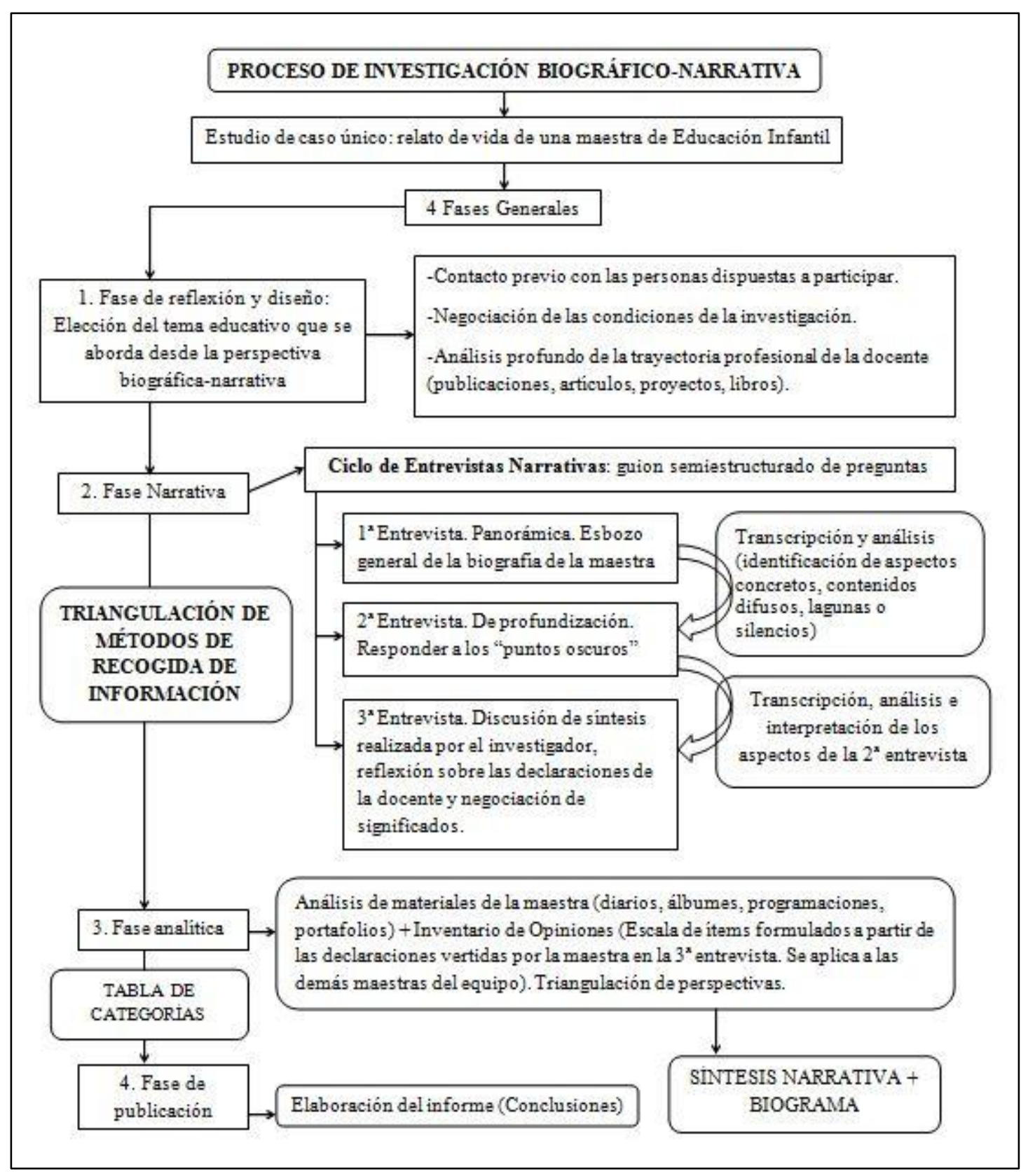

Gráfico 1. El proceso metodológico de la investigación biográfico-narrativa

b) El inventario de declaraciones. La tabla de categorías recogió cada una de las dimensiones desarrolladas en la entrevista y facilitó la estructuración de la información, con la finalidad de construir el inventario de declaraciones. Se optó por una escala Likert, un cuestionario formado por diferentes ítems en el que las encuestadas muestran su acuerdo o desacuerdo con el enunciado, tomando como base las declaraciones consensuadas con la maestra. La utilización de este instrumento permitió contrastar las opiniones de la docente con las de sus compañeras, contribuir a la objetividad de la investigación y completar los datos recogidos en el informe definitivo. La formulación inicial del borrador fue sometido a un proceso de análisis compartido por parte de cuatro docentes de la Universidad de Huelva a fin de verificar la claridad de los ítems e 
instrucciones. Se llevó a cabo una aplicación experimental del instrumento: cumplimentación, demanda de opinión (tiempo requerido, claridad de los enunciados, etc.). La versión definitiva del instrumento fue aplicado a las cinco maestras que conformaban, junto a la docente protagonista del relato, el equipo de ciclo.

c) Análisis de materiales personales de la profesora. Se han examinado diversos materiales personales de la maestra relacionados con su desarrollo profesional en los trabajos por proyectos: diario autobiográfico, álbumes de experiencias, programaciones, artículos publicados, fotografías, materiales de aula, entre otros. El procedimiento de análisis efectuado ha respondido a cuestiones importantes sobre su utilización, esto es, cómo se emplea, con qué finalidad, qué han aportado a su trayectoria profesional, cómo contribuye a mejorar su praxis docente (McMillan \& Schumacher, 2005).

Para alcanzar la validez y la fiabilidad del proceso de investigación, se han adoptado diferentes estrategias: la triangulación de métodos para integrar diferentes datos sobre la realidad profesional de la docente, la triangulación de perspectivas, de cara a completar la información personal del relato a partir de las aportaciones de otras voces y la búsqueda de consenso con la protagonista, alcanzando un acuerdo de significados implícitos en su narración.

\section{Resultados}

Tras el análisis exhaustivo de cada una de las categorías propuestas para el estudio, se han obtenido los siguientes resultados, estableciendo nexos comunes y diferencias significativas entre el punto de vista de la docente y del resto de compañeras respecto a cada uno de los temas abordados (ver Tabla 1).

Tabla 1

Tabla de categorías, preguntas de investigación y objetivos establecidos en el proceso de indagación biográfico-narrativa.

\begin{tabular}{|c|c|c|}
\hline CATEGORÍAS & PREGUNTAS & OBJETIVOS \\
\hline $\begin{array}{l}\text { 1. Etapa inicial de la } \\
\text { trayectoria } \\
\text { profesional EIT }\end{array}$ & $\begin{array}{l}\text {-¿Qué motivos condicionaron tu } \\
\text { decisión de estudiar Magisterio? } \\
\text { ¿¿Cómo influyó el periodo socio } \\
\text { histórico en tus comienzos? } \\
\text {-¿Existieron circunstancias familiares } \\
\text { que influyeran en tu decisión? }\end{array}$ & \multirow{2}{*}{$\begin{array}{l}\text { Interpretar el testimonio } \\
\text { subjetivo de la docente a } \\
\text { partir de las condiciones y las } \\
\text { circunstancias familiares, } \\
\text { personales, históricas, } \\
\text { sociales y pedagógicas que } \\
\text { han marcado su vida. }\end{array}$} \\
\hline $\begin{array}{l}\text { 2. Fase de tránsito al } \\
\text { trabajo estable } \\
\text { RTE }\end{array}$ & $\begin{array}{l}\text {-¿Cuándo accediste al puesto de } \\
\text { maestra funcionaria? } \\
\text { ¿¿Existen personas que fueran } \\
\text { importantes en este periodo? }\end{array}$ & \\
\hline $\begin{array}{l}\text { 3. Trabajo estable. } \\
\text { Primeras } \\
\text { experiencias y } \\
\text { Formación } \\
\text { permanente TPF }\end{array}$ & $\begin{array}{l}-¿ \text { ¿Cuál fue tu primer destino laboral } \\
\text { significativo? } \\
-¿ \text { ¿ué tipo de formación permanente } \\
\text { has recibido sobre Didáctica Infantil? } \\
-\succsim C \text { ¿ómo se diseñaron y desarrollaron } \\
\text { tus primeros proyectos? }\end{array}$ & $\begin{array}{l}\text { Construir la biografía } \\
\text { evolutiva de la maestra, } \\
\text { identificando las influencias, } \\
\text { los incidentes críticos, sus } \\
\text { reflexiones y el impacto de } \\
\text { otras vidas en su práctica. }\end{array}$ \\
\hline
\end{tabular}


Tabla 1 (Cont.)

Tabla de categorias, preguntas de investigación y objetivos establecidos en el proceso de indagación biográfico-narrativa.

\begin{tabular}{|c|c|c|}
\hline CATEGORÍAS & PREGUNTAS & OBJETIVOS \\
\hline $\begin{array}{l}\text { 4. Trabajo por } \\
\text { proyectos } \\
\text { TPR }\end{array}$ & $\begin{array}{l}-¿ \text { Cómo entraste en contacto con la } \\
\text { idea de trabajar por proyectos? } \\
\text {-¿Qué factores facilitaron y } \\
\text { obstaculizaron el desarrollo de los } \\
\text { proyectos de trabajo? ¿Cómo } \\
\text { abordas la educación intercultural en } \\
\text { los proyectos de trabajo? }\end{array}$ & $\begin{array}{l}\text { Estudiar en profundidad la } \\
\text { relación entre los proyectos de } \\
\text { trabajo y la } \\
\text { interculturalidad desde la } \\
\text { perspectiva biográfico-narrativa de } \\
\text { la maestra }\end{array}$ \\
\hline $\begin{array}{l}\text { 5. Madurez y } \\
\text { desarrollo } \\
\text { profesional TMD }\end{array}$ & $\begin{array}{l}-¿ \text { ¿De qué manera ha evolucionado los } \\
\text { trabajos por proyectos en tu } \\
\text { trayectoria profesional? } \\
-¿ \text { Cómo se lleva a efecto la } \\
\text { colaboración de las familias con los } \\
\text { proyectos de trabajo? }\end{array}$ & $\begin{array}{l}\text { Conocer y comprender el relato } \\
\text { de vida de la docente, cuya } \\
\text { madurez personal y profesional se } \\
\text { articulan en torno a los proyectos } \\
\text { de trabajo. }\end{array}$ \\
\hline $\begin{array}{l}\text { 6. Proceso de } \\
\text { enseñanza- } \\
\text { aprendizaje en } \\
\text { Educación Infantil } \\
\text { TEI }\end{array}$ & $\begin{array}{l}\text {-¿Cómo se lleva a cabo la } \\
\text { coordinación con el equipo de } \\
\text { Educación Primaria? } \\
\text { cambios crees que se deberían } \\
\text { introducir en la Educación Infantil? }\end{array}$ & $\begin{array}{l}\text { Describir la valoración personal } \\
\text { del equipo docente sobre las } \\
\text { declaraciones de la maestra acerca } \\
\text { del proceso de enseñanza- } \\
\text { aprendizaje en Educación Infantil. }\end{array}$ \\
\hline $\begin{array}{l}\text { 7. Proyección de } \\
\text { futuro PFU }\end{array}$ & $\begin{array}{l}\text { - ¿Cómo afrontas tu nueva etapa como } \\
\text { futura maestra de Educación Primaria? } \\
\text { ¿¿Qué elementos pueden facilitar su } \\
\text { desarrollo en Ed. Primaria? }\end{array}$ & $\begin{array}{l}\text { Explicar las expectativas y las } \\
\text { perspectivas futuras de la maestra } \\
\text { en su nueva proyección } \\
\text { profesional. }\end{array}$ \\
\hline
\end{tabular}

\section{Etapa inicial de la trayectoria profesional}

Cabe destacar que la maestra siente la vocación de dedicarse a la docencia desde muy temprana edad. Una de las personas que condicionó su decisión de optar por esta vía fue un profesor de francés que le impartió docencia durante su etapa formativa en Bachillerato. Le encantaba la metodología que empleaba este docente, su forma especial de enseñar, ya que marcó un punto de inflexión respecto al resto de profesionales. Rompía con la rutina de lecciones teóricas y realizaba más actividades prácticas. Esta es la principal razón que justifica su elección de estudiar Magisterio de Lengua Castellana-Francés. En este sentido, Goodson (2009, p.748) advierte que "una de las características más comunes que hacen los docentes sobre sus antecedentes es la presencia de un profesor favorito que influyó en ellos como jóvenes alumnos." Si se compara la importancia que concede la entrevistada al factor vocacional para ser maestra de Educación Infantil, con aquella relevancia otorgada por sus compañeras de equipo, se pueden apreciar diferencias poco notables. Un aspecto positivo que ella destaca de su formación inicial es la solidez de los conocimientos, que le han aportado un bagaje cognitivo nada desdeñable que ha aprendido a valorar en su experiencia profesional. El período histórico que enmarcó su trayectoria universitaria fue la Transición española (1975-1978), que abrió nuevos cauces a una pedagogía más innovadora, rompiendo con las bases establecidas anteriormente y bridando la posibilidad de realizar nuevos proyectos. Ella depositó su confianza en la nueva época, que vendría llena de oportunidades para mejorar la educación: "Yo 
recuerdo aquella época con mucha ilusión por hacer cosas nuevas, porque todo lo que iba a venir detrás era mucho mejor. Ese es el recuerdo que yo tengo" (EIT, Entrevista 01, Pregunta 12).

\section{Fase del tránsito al trabajo estable}

En primera instancia, conviene subrayar que ella accedió a su primer puesto de maestra funcionaria en 1988 mediante oposiciones del Estado. Reconoce que le costó bastante esfuerzo aprobarlas, pero pronto tomó conciencia que ésta era la única vía para acceder a un puesto de trabajo definitivo. A pesar de que su especialidad era de Lengua-Castellana Francés, optó por presentarse a la especialidad de Educación Infantil, ya que apenas se convocaban plazas para cubrir los puestos vacantes de maestra de francés. Otros factores decisivos fueron las experiencias previas en esta etapa educativa, mientras sustituía en el colegio.

Cuando obtuvo su plaza de maestra funcionaria, comenzó a participar en proyectos educativos relacionados con la innovación metodológica, la colaboración de seminarios, los talleres de formación permanente y las escuelas de verano. Su mayor deseo era renovar una escuela que carecía de "motivación, interés, entusiasmo. Yo la veía más bien aburrida. La veía aburrida, monótona, machacona” (RTE, Entrevista 01, pregunta 22). Reconoce que la asunción de estas ideas renovadoras fue el principal motivo para empezar a asistir por iniciativa propia a diferentes cursos y talleres de formación, ya que ella quería ofrecer una respuesta individualizada a su alumnado y ampliar los conocimientos teóricos que había adquirido en la Universidad. Sus compañeras de equipo no muestran la misma opinión, manifestando su acuerdo con la validez de la formación didáctica que ellas recibieron en la universidad para desarrollar actividades adaptadas a las características de sus alumnos y alumnas. Por consiguiente, ellas no han sentido tanto la necesidad de formarse posteriormente porque contaban con una buena formación inicial universitaria.

\section{Trabajo estable: Primeras experiencias y Formación permanente}

Su destino laboral más relevante fue el Colegio Público "Gaviota”, situado en el municipio de Punta Umbría. Ella lo recuerda como su época más feliz, porque la organización escolar de este centro educativo y la manera de trabajar se aproximaban más a su concepción progresista de la enseñanza. Desde esta perspectiva, la maestra asevera que el grupo de compañeros y compañeras era muy compacto, mantenían la misma ideología sobre la pedagogía y unos pensamientos convergentes hacia la misma dirección: una docencia renovada. Los aspectos positivos a destacar son la oportunidad para acometer iniciativas y la ilusión por comenzar a trabajar de otra manera, mientras que los elementos negativos son la excesiva confianza en la improvisación propia de la inexperiencia y la carencia de reflexión sobre la propia práctica. La maestra admite que "aquella época era más activa, más de hacer muchas actividades: todas muy motivadoras e interesantes" (TPF, Entrevista 03, pregunta 11). Su extensa formación permanente se caracteriza por la estrecha relación que guarda con la innovación curricular como motor de cambio y por la educación emocional en la etapa de Educación Infantil.

\section{Hacia un aprendizaje significativo. Los trabajos por proyectos}

En el año 2001, con la plaza definitiva en el CEIP Virgen del Carmen (Punta Umbría), la maestra entró en contacto por primera vez con los trabajos por proyectos a partir de una propuesta de la Universidad de Huelva. El Grupo Andaluz de Investigación en el Aula (GAIA) le brindó la oportunidad de participar en un proyecto de investigación escolar. Cabe destacar que ella trabajaba con propuestas globalizadas similares en su anterior destino laboral, el Colegio Gaviota, a pesar de 
que las denominaban "Unidades Didácticas", articuladas en torno a los centros de interés. A modo de comparación, ella declara: "el trabajo por proyectos es un trabajo que requiere más reflexión, más elaboración, más investigación. Es más investigativo y está más organizado” (TPR, Entrevista 01, pregunta 38).

Los elementos que facilitaron el desarrollo de los trabajos por proyectos fueron la formación permanente adquirida por la maestra, a través de lecturas específicas, y el intercambio de opiniones con otras compañeras de su equipo. Los principales obstáculos estaban relacionados con la falta de apoyo profesional por parte de algunas docentes y la escasez de tiempo para investigar. La maestra ratifica que "el reparto de responsabilidades es fundamental. Si tú no lo tienes, si tú estás sola, evidentemente, eso es un obstáculo bastante grande” (TPR, Entrevista 01, Pregunta 50).

\section{La experiencia y la práctica reflexionada. Madurez y desarrollo profesional}

La maestra intenta incorporar la realidad intercultural de su aula en los trabajos por proyectos, de forma que exista una permeabilidad entre el tema y las diferentes aportaciones que la diversidad cultural le proporciona: "Siempre intento incluir los aspectos culturales de otros sitio para que los niños conozcan que hay otras formas de escribir, expresarse y comer, que son iguales que las nuestras pero en sus países" (TMD, Entrevista 01, pregunta 55). Defiende que la educación intercultural, a través de los trabajos por proyectos, se aborda de manera más global que mediante otro modelo de desarrollo curricular. Dos maestras encuestadas $(33,33 \%)$ están totalmente de acuerdo con el enunciado, seguido de un porcentaje similar que está de acuerdo. No obstante, una encuestada (16,67\%) no se muestra de acuerdo ni en desacuerdo, mientras que la otra está en desacuerdo y piensa que la educación intercultural se puede abordar de manera más holística bajo enfoques diferentes a los proyectos de trabajo.

\section{La identidad de la Educación Infantil. Proceso de enseñanza-aprendizaje}

La maestra reconoce de forma expresa que "si se imparten Infantil y Primaria en la misma escuela, siempre hay miras a conseguir que el niño esté preparado para entrar en Primaria" (TEI, Entrevista 03, pregunta). Ello explica que los cambios que debería experimentar la Educación Infantil, en su opinión, estén orientados al establecimiento de un pequeño período de adaptación desde esta etapa a la Educación Primaria. En dos meses (el tiempo que comprende el período estival) cambia de forma radical la metodología y la didáctica de la nueva etapa, pero el alumnado no presenta grandes avances en el desarrollo de sus capacidades (por ejemplo: la adquisición de autonomía en sus actividades cotidianas, las habilidades lógico-matemáticas, en lectoescritura y en diferentes lenguajes). Esta declaración lleva aparejada la reclamación de un mayor reconocimiento hacia el aprendizaje que adquiere el alumnado en Educación Infantil, defendiendo que los profesionales de Educación Primaria deben acercarse más a los maestros de Educación Infantil y mostrar una actitud comprensiva hacia su quehacer educativo.

Uno de los elementos necesarios en la Educación Infantil es la incorporación de un mayor número de profesionales. La profesora insiste en el requerimiento de más de un docente en el aula, puesto que "cuanto más pequeños son los niños, más necesitan un aprendizaje individualizado porque tienen poca autonomía. Hay cosas que tú puedes hacer en grupos pequeños. Si tienes 25 alumnos, formas cinco grupos pequeños" (TEI, Entrevista 03, Pregunta 27). 


\section{Proyección de futuro. Fortalezas y retos ante las puertas de una nueva etapa}

Las primeras sensaciones que invaden a la profesora son de incertidumbre, recelo y presión porque, en sus palabras: "paso a una etapa donde hay mucha presión social a causa de las pruebas externas que le hacen a los niños. Los contenidos son tan importantes que los pobrecitos están todo el día haciendo exámenes" (PFU, Entrevista 01, pregunta 67). Este es el principal motivo por el cual está realizando cursos de innovación educativa dirigidos a docentes de Educación Primaria. La formación constituye la mejor garantía de adaptación para dar respuesta a las necesidades de su futuro alumnado. Algo que le preocupa sobremanera es la excesiva utilización de los libros de textos en esta etapa como único recurso. Ante el ítem "En la Educación Primaria, los docentes tienen más presión familiar que en la Educación Infantil, ya que están más condicionados por las pruebas de evaluación", tres maestras encuestadas afirman estar de acuerdo y otras dos no muestran acuerdo ni desacuerdo. Sólo una de ellas está en desacuerdo con el enunciado.

Los principales elementos facilitadores con los que cuenta para desarrollar los proyectos de trabajo en la Educación Primaria son: la búsqueda de contactos con otras maestras que trabajan bajo este enfoque en centros educativos próximos (con la intención de crear una red de apoyo docente e intercambio de experiencias) y la actitud positiva de algunos compañeros de Educación Primaria, a partir del conocimiento adquirido sobre prácticas relacionadas con esta modalidad de desarrollo curricular.

\section{Discusión y conclusiones}

La formación inicial de la maestra estuvo marcada por el entramado de tres factores: la pasión por la docencia, el carácter teórico de los conocimientos pedagógicos adquiridos en la Universidad y el período histórico de la Transición española, dejando paso a una etapa menos conservadora orientada al cambio. Se puede apreciar como la confluencia de estos tres elementos conforma una realidad consustancial a la trayectoria de la maestra, de manera que cada uno ejerce una influencia sobre el resto.

Como advierten R. Butt, D. Townsend y D. Raymond (Marcelo \& Mingorance, 1992, p. 206): "El desarrollo del profesor se ha vinculado estrechamente al contexto político, social y económico de la enseñanza". Se percibe a todas luces como la transmisión excesivamente teórica de los contenidos adquiridos por la maestra (independiente de su aplicación en la práctica y, por consiguiente, lejos de la realidad educativa) se hallaba en las antípodas de su pensamiento innovador. El razonamiento hacia esta dirección promovió su interés hacia la lectura de autores relacionados con la educación progresista (Célestin Freinet, Ovide Decroly, María Montessori, entre otros). Se debe tener presente que la reflexión forma parte del aprendizaje de los profesores, contribuyendo a su vez a liberarles de la conducta impulsiva y las rutinas. De este modo, empiezan a actuar de forma intencionada para lograr ciertos propósitos (Day, 2006).

Salgueiro (1997) señala que el conocimiento y la reflexión sobre la práctica deberían incorporarse a los cursos de formación, con vistas a disminuir la distancia entre las teorías pedagógicas inherente a éstos y la realidad cotidiana del profesorado.

Una idea no menos trascendental aparece vinculada a los motivos que desencadenaron la decisión de la maestra para trabajar por proyectos, convirtiéndose en adepta de este planteamiento curricular. Su primera inmersión en el ambiente de los proyectos fue en 2001, a partir de una propuesta de GAIA, que representó una oportunidad para satisfacer sus deseos de abordar temas abiertos y actuales con su alumnado, partiendo de sus intereses. Dos elementos facilitadores a destacar son el apoyo de su marido y el intercambio de experiencias con otras compañeras de 
profesión. Los principales obstáculos fueron la carencia de apoyo de algunas compañeras y, por consiguiente, de la cultura de colaboración. En este sentido, Andy Hargreaves (2005, p.218) alega que "las relaciones de trabajo en colaboración no surgen a partir de ninguna limitación o imposición administrativa, sino del valor que los profesores les reconocen, derivado de la experiencia."

Hace cinco años, cuando dos alumnos de otras nacionalidades coincidieron en su curso, ella empezó a incorporar plenamente la realidad intercultural del aula en los proyectos. Existen motivos plausibles para lograr este propósito: el respeto a los conocimientos previos, los intereses y las necesidades de los niños de culturas diversas, la conexión de estas tres fuentes de conocimientos con los contenidos del currículum, el fomento del trabajo en equipo, el uso de las tecnologías de la información y la comunicación (TIC) con fines investigativos y la reflexión sobre asuntos de justicia social.

Las sensaciones más sobresalientes de la maestra, ante su incorporación a la Educación Primaria, son de incertidumbre, inquietud y presión, de manera que vaticina algunos obstáculos en la implantación de los trabajos por proyectos en la etapa, tales como la oposición de algunos familiares y de ciertos compañeros. En beneficio de su desarrollo, cuenta con razones favorables, destacando el intercambio de experiencias con educadoras de centros cercanos y la formación sobre innovación educativa que ha recibido en los últimos meses. Por unanimidad, las personas encuestadas muestran su acuerdo con el uso exclusivo de las editoriales en la Educación Primaria como recurso educativo, restando mucha autonomía profesional al docente. Day (2012) afirma que ser y comportarse como un profesional, en el sentido de ejercer un grado de autonomía y demostrar compromiso con la educación de los alumnos, es una expectativa. Los libros de textos ocupan un lugar preeminente en muchos niveles educativos, condicionando los contenidos, la forma de impartirlos, el orden sucesivo de las unidades didácticas y el tipo de evaluación (Gerver, 2012). De acuerdo con Torres (2012, p. 180): "En la medida en que en este recurso se encuentra lo que cada estudiante debe recordar, éstos podrían dejar de ir al centro escolar y dedicarse a memorizar tales libros, con lo que el profesorado estaría de más".

\section{Referencias}

Apple, M., \& Beane, J. (2005). Escuelas democráticas. $4^{a}$ Edición. Madrid: Morata.

Buendía, L., Colás, P., \& Hernández Pina, F. (1998). Métodos de investigación en psicopedagogía. Madrid: Mc Graw-Hill.

Bolívar, A., Domingo, J., \& Fernández, M. (2001). La investigación biográfico-narrativa en educación: enfoque y metodología. Madrid: La Muralla.

Day, C. (2006). Pasión por enseñar. La identidad personal y profesional del docente y sus valores. Madrid: Narcea.

Day, C. (2012). Profesores: Vidas nuevas, verdades antiguas. Madrid: Narcea.

Díez, C. (2002). La oreja verde de la escuela. Trabajo por proyectos y vida cotidiana en la escuela infantil. Madrid: Ediciones de la Torre.

Feito, R. (2006). Los contenidos curriculares en una escuela de primaria innovadora.Entre lo previsto y lo improvisado. Revista de Educación, 340, 1147-1169.

Gerver, R. (2012). Crear hoy la escuela del mañana. La educación y el futuro de nuestros hijos. Madrid: Ediciones SM.

Goodson, I. (2003). Hacia un desarrollo de las historias personales y profesionales de los docentes. Revista Mexicana de Investigación Educativa, 8(19), 733-758. 
Guarro, A. (2005). La transformación democrática de la cultura escolar: Una respuesta justa a las necesidades del alumnado de zonas desfavorecidas. Profesorado, revista de currículum y formación del profesorado, 9, 1-48.

Hargreaves, A. (2005). Profesorado, cultura y postmodernidad. Cambian los tiempos, cambia el profesorado. $5^{a}$ Edición. Madrid: Morata.

Hernández, F., \& Ventura, M. (2008). La organización del currículum por proyectos de trabajo. Barcelona: Octaedro.

Kvale, S. (2011). Las entrevistas en investigación cualitativa. Madrid: Morata.

López Melero, M., \& Parages López, M. (2012). Para poder trabajar por proyectos de investigación en el aula, primero debemos conocernos. Revista de Educación Inclusiva, 5 (1), 83-94.

Marcelo, C., \& Mingorance, P. (1992). Pensamiento de profesores y desarrollo profesional. Sevilla: Servicio de Publicaciones de la Universidad de Sevilla.

Martínez Bonafé, J. (2002). Políticas del libro de texto escolar. Madrid: Morata.

Mcmillan, J., \& Schumacher, S. (2005). Investigación educativa. Una introducción conceptual. $5^{a}$ Edición. Madrid: Pearson.

Pérez Gómez, A. (1998). La cultura escolar en la sociedad neoliberal. Madrid: Morata.

Pérez Serrano, G. (2001). Investigación cualitativa. Retos e interrogantes I. Métodos. $3^{a}$ Edición. La Muralla: Madrid.

Pozuelos, F. (2007). Trabajo por proyectos en el aula: Descripción, investigación y experiencias. Sevilla: Cooperación educativa.

Salgueiro, A. (1997). La práctica docente cotidiana en el aula: el proceso de negociación. Investigación en la escuela, 31, 63-71.

Salgueiro, A. (1998). Saber docente y práctica cotidiana. Barcelona: Octaedro.

Simons, H. (2011). El estudio de caso: Teoría y práctica. Madrid: Morata.

Torres, J. (2012). Globalización e interdisciplinariedad: el currículum integrado. $\sigma^{a}$ Edición. Madrid: Morata.

\section{Sobre el Autor}

Autor: Pablo Javier Ortega Rodríguez.

Institución: Departamento de Educación. Facultad de Ciencias de la Educación. Campus El Carmen. Avda. Tres de Marzo, s/n, 21071 Huelva.

E-mail: pablojavier.ortega@alu.uhu.es

Información biográfica: Diplomado en Magisterio de Lengua Extranjera (Francés) (2009). Licenciado en Psicopedagogía (2012). Máster Oficial en Educación Especial (2012). Máster Oficial en Educación Intercultural (2013). Doctorando del Programa Oficial de Doctorado en Ciencias Sociales y de la Educación.

ORCID: no disponible 


\section{Investigación en la Escuela}

Revista académica evaluada por pares y de acceso abierto

Número 88

1 de octubre 2016

ISSN 2443-9991

\section{@}

SOMERIIGHISRESEEVED Los/as lectores/as pueden copiar, mostrar, y distribuir este artículo, siempre y cuando se de crédito y atribución al autor/es y a Investigación en la Escuela, se distribuya con propósitos no-comerciales, no se altere o transforme el trabajo original. Más detalles de la licencia de Creative Commons se encuentran en http://creativecommons.org/licenses/by-nc-sa/3.0 Cualquier otro uso debe ser aprobado en conjunto por el autor/es, o Investigación en la Escuela.

Contribuya con comentarios y sugerencias en la web de la revista. Por errores y sugerencias contacte a investigacionescuela@,ddcc.uhu.es 


\section{Investigación en la escuela}

Consejo de dirección: Ana Rivero García (Universidad de Sevilla), Nicolás de Alba Fernández (Universidad de Sevilla), Pedro Cañal de León (Universidad de Sevilla), Francisco F. García Pérez (Universidad de Sevilla), Gabriel Travé González, (Universidad de Huelva), Francisco F. Pozuelos Estrada (Universidad de Huelva)

\section{Dirección: Ana Rivero García y Nicolás de Alba Fernández}

Técnico de edición: Francisco Javier López Sánchez

\section{Consejo editorial}

José Félix Angulo Rasco. Universidad de Cádiz Rosa Ma Ávila Ruiz. Universidad de Sevilla Pilar Azcárate Goded. Universidad de Cádiz Juan Bautista Martínez Rodríguez. Universidad de Granada

Nieves Blanco García. Universidad de Málaga Fernando Barragán Medero. Universidad de La Laguna José Carrillo Yáñez. Universidad de Huelva José Contreras Domingo. Universidad de Barcelona. Luis C. Contreras González. Universidad de Huelva Ana $\mathbf{M}^{\mathbf{a}}$ Criado García-Legaz. Universidad de Sevilla Rosario Cubero Pérez. Universidad de Sevilla José $\mathbf{M}^{\mathbf{a}}$ Cuenca López. Universidad de Huelva Jesús Estepa Giménez. Universidad de Huelva Rafael Feito Alonso. Universidad Complutense (Madrid)

Francisco José García Gallardo. Universidad de Huelva

Soledad García Gómez. Universidad de Sevilla J. Eduardo García Díaz. Universidad de Sevilla
Fernando Hernández Hernández. Universidad de Barcelona

Salvador Llinares Ciscar. Universidad de Alicante Alfonso Luque Lozano. Universidad de Sevilla Rosa Martín del Pozo. Universidad Complutense (Madrid)

José Martín Toscano. IES Fernando Herrera (Sevilla) Jaume Martínez Bonafé. Universidad de Valencia F. Javier Merchán Iglesias. Universidad de Sevilla Emilia Moreno Sánchez. Universidad de Huelva. Rosario Ortega Ruiz. Universidad de Córdoba Antonio de Pro Bueno. Universidad de Murcia Fco. de Paula Rodríguez Miranda. Universidad de Huelva

Pedro Sáenz-López Buñuel. Universidad de Huelva Antoni Santisteban Fernández. Universidad Autónoma (Barcelona)

Emilio Solís Ramírez. Catedrático de IES. $\mathbf{M}^{\mathbf{a}}$ Victoria Sánchez García. Universidad de Sevilla. Magdalena Suárez Ortega. Universidad de Sevilla

\section{Consejo asesor}

Manuel Area Moreira. Universidad de La Laguna

Jaume Carbonell. Director Cuadernos de Pedagogía. Barcelona

César Coll. Universidad de Barcelona

Christopher Day. Universidad de Nothingham. U.K.

Juan Delval. Universidad Nacional de Educación a Distancia

John Elliott. Universidad de East Anglia. Norwich. U.K.

José Gimeno Sacritán. Universidad de Valencia

André Giordan. Universidad de Paris VII y Ginebra

Francisco Imbernón. Universidad de Barcelona

Ángel Pérez Gómez. Universidad de Málaga

Rafael Porlán Ariza. Universidad de Sevilla

Francesco Tonucci. Instituto de Pedagogía del C.N.R. Roma

Jurjo Torres Santomé. Universidad de A Coruña 